\title{
Improving Noun Hyponyms Using Select TED Wordlist through WordNet: An Investigational Study
}

\author{
Aravind B R*, Rajasekaran V \\ Division of Social Sciences \& Languages, Vellore Institute of Technology, India
}

Received September 16, 2019; Revised October 21, 2019; Accepted October 28,2019

Copyright $\bigcirc 2019$ by authors, all rights reserved. Authors agree that this article remains permanently open access under the terms of the Creative Commons Attribution License 4.0 International License

\begin{abstract}
Vocabulary teaching is one of the most imperative aspects of instruction for ESL learners because it influences both productive and respective skills of students. This prudent study stresses and explores how to use hyponyms of the select TED wordlist nouns in academic writing. Therefore, the qualitative gap in vocabulary understanding of ESL learners can be linked. The method made use of WordNet to evaluate and the outcome was analyzed. The acquired results of this research revealed that the effective use of WordNet increased more acquaintance for learning the meaning of new vocabulary.
\end{abstract}

Keywords Vocabulary Skills, TED Wordlist, ESL Learners, Wordnet, Hyponyms, Vocabulary Acquisition

\section{Introduction}

Vocabulary knowledge is a fundamental ingredient of knowing any foreign language. Vocabulary familiarity sharpens both communication and comprehension skills. Poor vocabulary-based skills of ESL learners lead to the loss of many job opportunities (Roe, 2016). Vocabulary richness influences students' receptive and productive skills. Therefore, writing, reading, listening and speaking skills can be enhanced through the development of students' vocabulary. Given that English is a lingua franca, learning English vocabulary is part of knowledge acquisition in various areas of study (Alqahtani, 2015). In pedagogy literature, there were three main strategies dedicated to teach vocabulary in classrooms: the keyword method (Atkinson, 1975), semantic processing method (Beck et al., 1987) and contextual processing (Sternberg, 1987). Because of the centrality of English vocabulary to learning ESL, several methods have been introduced to facilitate vocabulary acquisition.

\subsection{TED and WordNet}

TED talks were used as an annexure towards a power pointed management to make a demonstration anywhere (Ramanayake \& Williams, 2017). TED, or Technology Entertainment and Design, was started in 1984 and covers all the topics on Earth and above in various cultures of the world (Roe, 2016). Electronic databases of English are large stores of English words structured in different ways based on several theoretical grounds. WordNet (WN), for instance, organizes English words according to their parts of speech. Therefore, WN has been used in several vocabulary-related tasks. WN is used to automatically construct thesauri because of its rich synonymy-based entries (Mandala et al., 1998), disambiguate the senses of polysemous nouns and verbs (Moldovan \& Mihalcea, 2000), improve the writing performance of writers using ESL (Resnick, 1993) and perform vocabulary-based assessment of learners’ proficiency level (Hu \&Graesser, 1998).

\subsection{Hyponyms and WordNet}

In linguistics, a hyponym is a word or phrase whose semantic field is included within that of another word, its hyperonym or hypernym. In simpler terms, a hyponym is in a type-of relationship with its hypernym. For example, pigeon, crow, eagle and seagull are all hyponyms of bird; which, in turn, is a hyponym of animal. WN, however, labels the semantic relations among words, whereas the groupings of words in a thesaurus do not follow any explicit pattern other than intuitive meaning similarity.

\section{Survey of Literature}

This section reviews some of these studies which are closely related to the current study. The ESL literature contains a number of studies which used corpora and WordNet to improve the vocabulary of ESL learners. 
Certain factors need to be considered like the society, grammar, culture etc. and that are required to be known and adopted in the vision of following any languages (Ramanayake \& Williams, 2017). TED serves as an exploratory factor for ESL learners' vocabulary knowledge through social factor (Aravind \& Rajasekaran, 2018). TED influences ESL learners' persuasive and argumentative vocabulary for effective communication (Aravind \& Rajasekaran, 2019). TEDs were utilized properly and they may help students to improve comprehending qualities in addition as an apparatus to learn in a much easy way (Massing et al., 2013). On the one hand, scholars exploited the structured lexicon of WordNet (WN) to provide non-native English learners with vocabulary-dependent knowledge. Lin (1997) used WN in vocabulary teaching because of the rich semantic relations that are used to define words and place them together. The study argued that teaching vocabulary fundamentally requires teaching students how to realize distinctions between related words. Therefore, an advanced thesaurus is needed to help students learning the meaning of a new word with relevance to its companions so that students can build their own semantic network. To perform this task, $\mathrm{WN}$, as a language resource, is used because of its rich variety of semantic relations. On the other hand, corpora are evidently beneficial in ESL learning tasks.

\section{Significance of the Study}

The current study contributes to the literature of applied linguistics and ESL. Given the implication of vocabulary learning to productive and receptive skills of ESL learners, the results of this study should improve English vocabulary. Moreover, this study initiates the learners to know semantic differences between synonymous nouns and verbs to accurately use them in academic writings. On the other hand, learners will benefit master the lexicographic skills about semantic relations of corpus-driven list of words. Implications on the design and teaching of pedagogical curricula can be made.

\section{Research Objectives}

This study accomplishes qualitative objectives. The present study aims:

- To inculcate ESL learners select TED wordlist nouns used by native speakers in academic writing.

- To alert and inform ESL learners the distinctions between hyponyms of the select TED wordlist nouns and its usage.

\section{Research Questions}

The existing study challenges to answer the following research questions. The first question is concerned with the applicability of TED corpus in vocabulary teaching. The second one explores the effectiveness of WordNet in teaching ESL learners the fine distinctions among hyponyms and its usage.

1. How effective are TED corpus in linking the quantitative gap between the enlarging vocabulary of native English speakers and that of ESL learners?

2. How WordNet enables learners to acquire hyponyms of select nouns from TED Corpus?

\section{Research Methodology}

The TED corpus contains one thousand seven hundred and seventy, TED Talk language transcriptions, started in the year 2006 and extended till 2014. TED website endures to stake new-fangled discussions each workaday. Then the production got improved as TED Word List (Ritchie \& Lewis, 2003). Finally, it was resulted that the proportion of TED Word List in the TED corpus and in what way the TED Word List augmented the whole attention of the terminology comprised on the General Service List, Academic Word List, and TED Word List (Kamil \& Hiebert, 2005). Participants' knowledge about the hyponyms of the nouns is evaluated and recorded. 10 questions were randomly sorted and distributed using Google forms. The correction of collected answers was automated. WordNet was used to generate the hyponyms of retrieved nouns.

\section{Research Samples and Sample Size}

To conduct this research study, a convenience sample was chosen consisting 36 ESL learners (23 males, 13 females) who were studying engineering in VIT Chennai, as shown in Table 1. The samples' proficiency level ranges between intermediate and advanced level. Participants' demographical details, length of time learning English and other background information were collected from the participants' responses.

Table 1. Description of samples

\begin{tabular}{|c|c|}
\hline Category & Number \\
\hline Male & 23 \\
\hline Female & 13 \\
\hline Total & 36 \\
\hline
\end{tabular}

\section{Procedure}

In order to improve ESL learners' hyponyms usage in academic writings, the current study made use of select TED wordlist nouns and WordNet. Initially, the learners were instructed to answer the questions asked in the Google forms genuinely by choosing the most accurate meaning of the 10 given nouns from the TED wordlist. After responding to the pre-test, the researcher collected 
the test-takers responses. At this point, the researcher gave the intervention of utilizing the WordNet by giving nouns randomly from the TED wordlist. The learners were made to practice in such a way to productively use WordNet and its unique features to acquire the knowledge about hyponyms. Finally, the researcher conducted the post-test by just giving the definition of ten different TED wordlist nouns to identify the appropriate hyponyms of the particular definition asked in the question. The reverse technique helped the researcher to know that learners' learnt to identify the most accurate and appropriate hyponyms without any choices and guesses.

Table 2. Select TED wordlist nouns for pre-test

\begin{tabular}{|c|}
\hline Words given in pre-test \\
\hline Ad \\
\hline Alien \\
\hline Bang \\
\hline Cable \\
\hline Drug \\
\hline Email \\
\hline Frog \\
\hline Tank \\
\hline Virus \\
\hline Web \\
\hline
\end{tabular}

Table 3. Select TED wordlist nouns for post-test

\begin{tabular}{|c|}
\hline Words expected in post-test \\
\hline Ant \\
\hline Autism \\
\hline Battery \\
\hline Carbon \\
\hline Dolphin \\
\hline Einstein \\
\hline Fuel \\
\hline Tag \\
\hline Video \\
\hline Website \\
\hline
\end{tabular}

\section{Result and Discussion of the Study}

The researcher endeavored to report the results and findings on the basis of the research objectives, to inculcate ESL learners' select TED wordlist nouns used by native speakers in academic writing and to alert and inform ESL learners the distinctions between hyponyms of the select TED wordlist nouns and its usage.

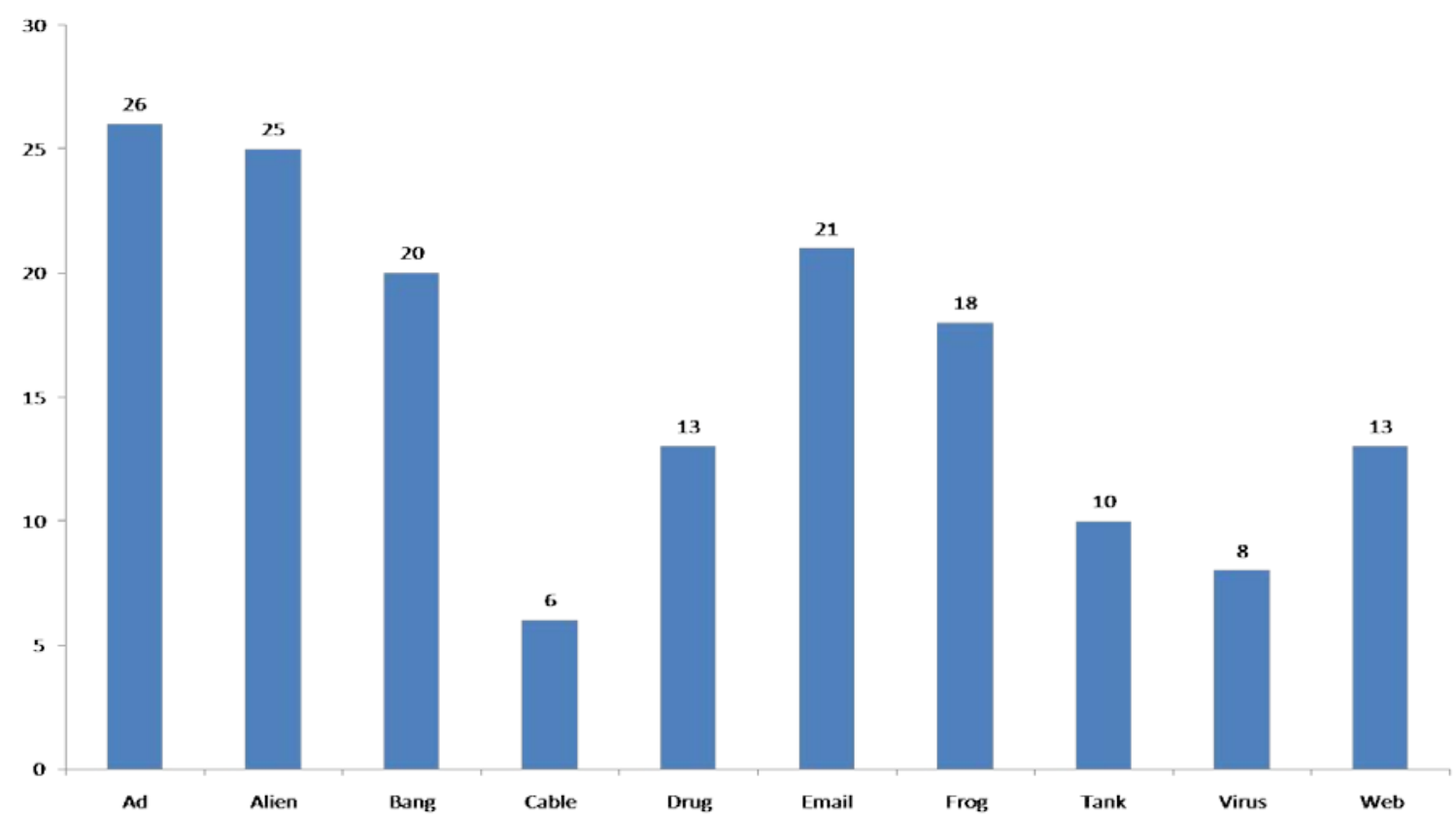

Figure 1. Graphical Representation of ESL Learners' Pre-test 


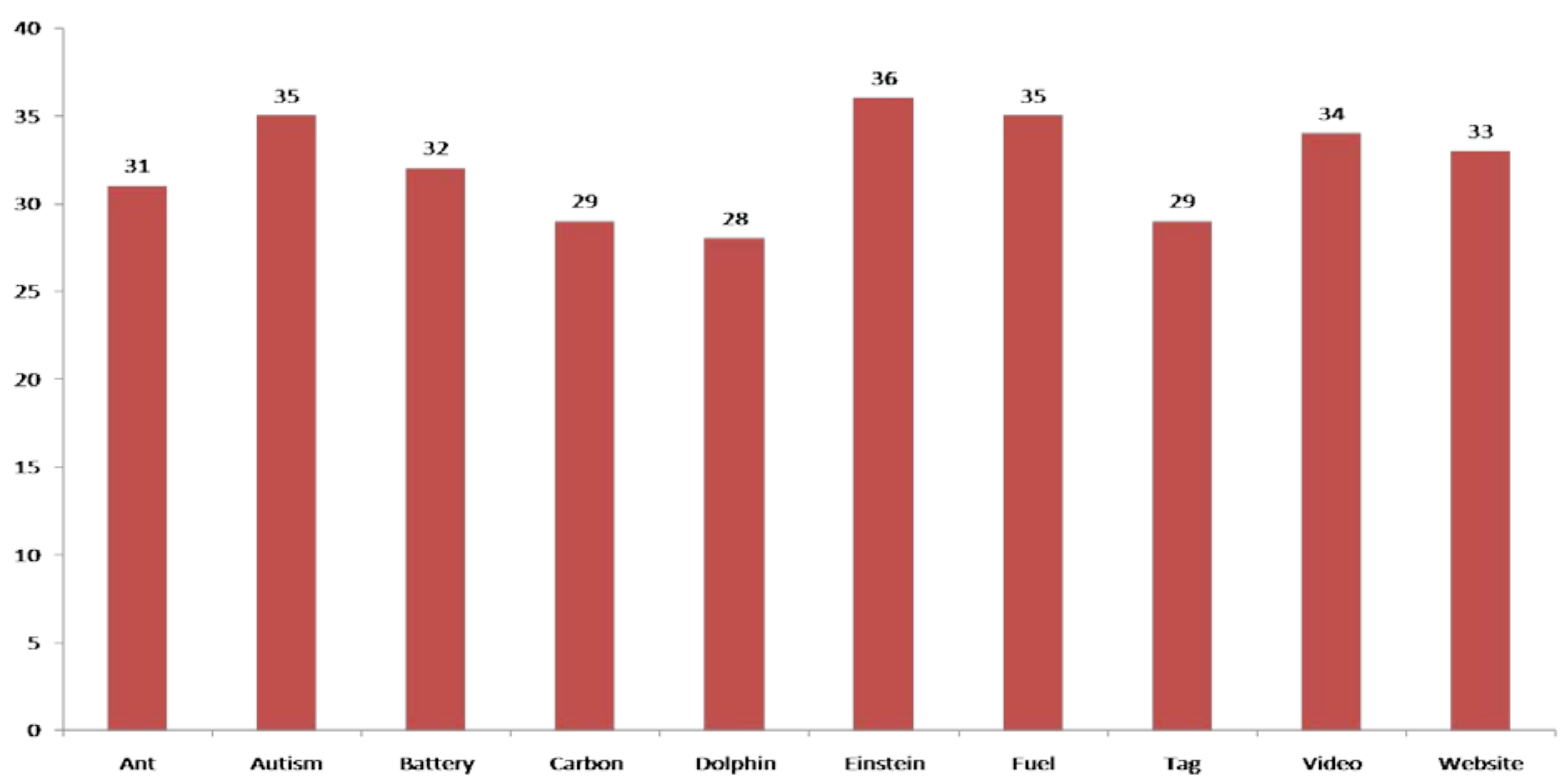

Figure 2. Graphical Representation of ESL Learners' Post-test

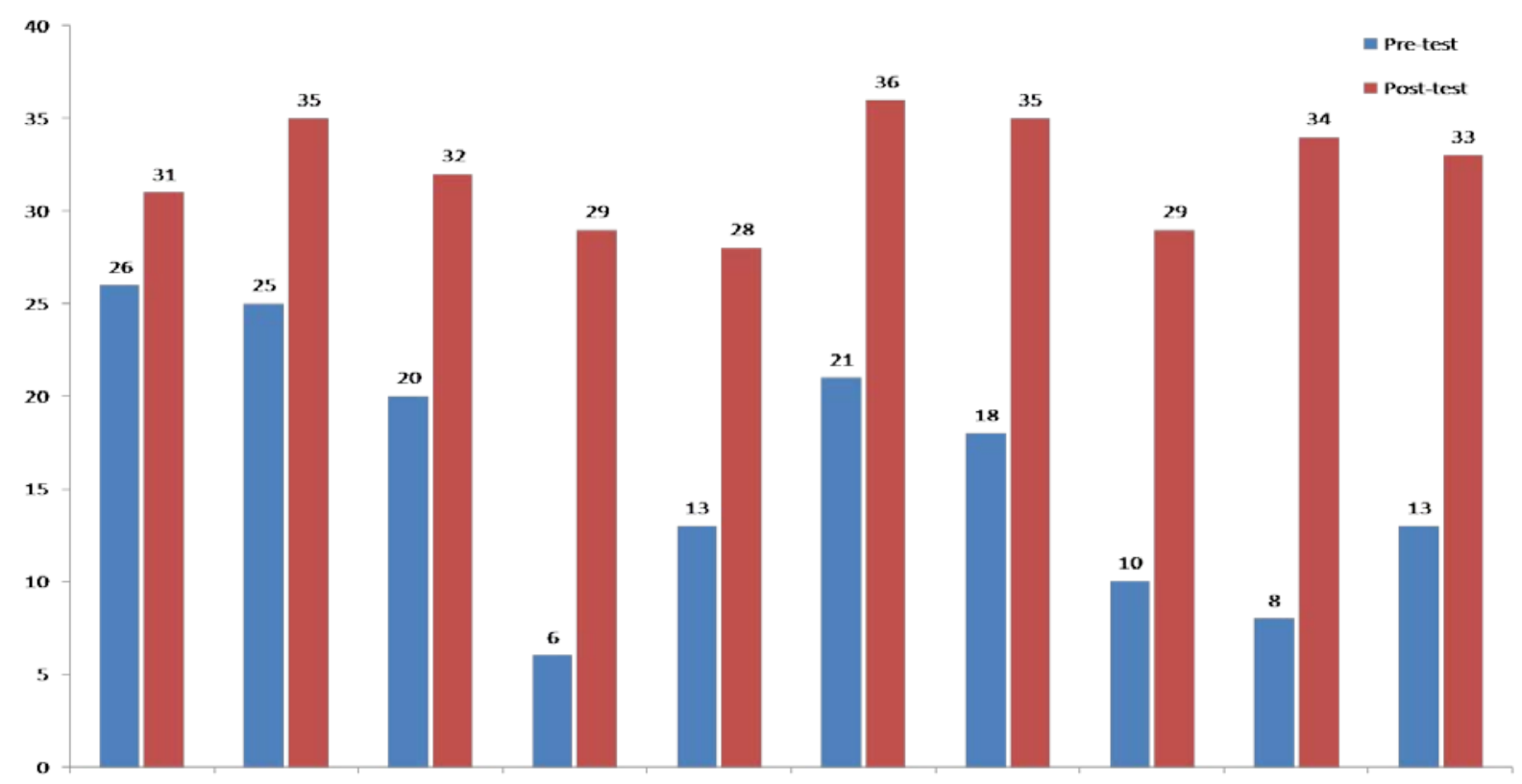

Figure 3. Graphical Representation of ESL Learners’ Pre and Post-test Comparative Results 


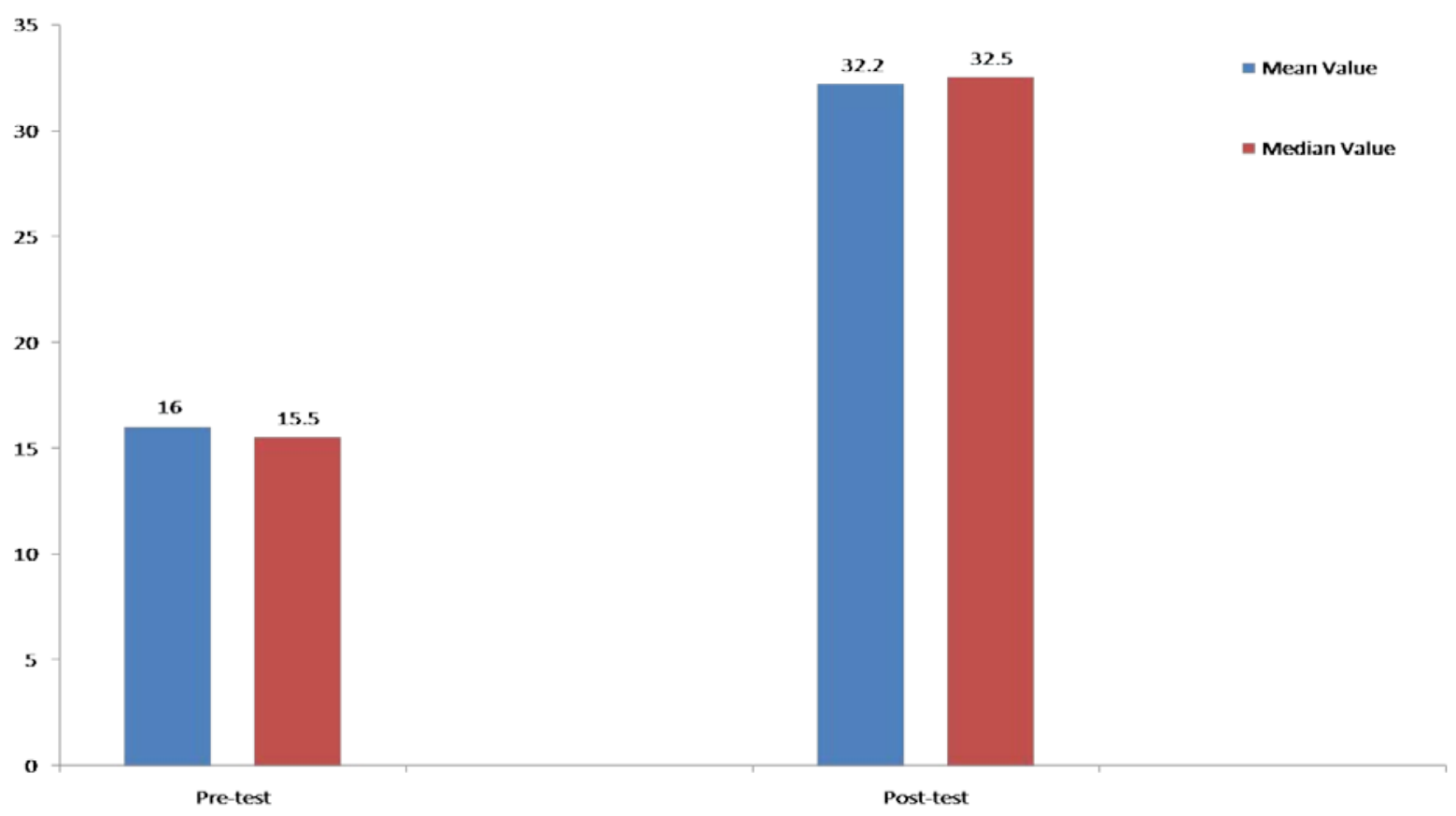

Figure 4. Graphical Representation of ESL Learners' Pre and Post-test Mean and Median Values from the Results

Analyzing the results of this investigational study, it has been observed that the existing study constitutes solution towards vocabulary skills qualitatively. The two experiments revealed that vocabulary knowledge and acquisition has significantly increased in the study. The pre-test posted select TED wordlist nouns like Ad, Alien, Bang, Cable, Drug, Email, Frog, Tank, Virus and Web, in order to know the learners' knowledge on hyponyms. The mean and median values of the pre-test are 16 and 15.5 respectively. The respondents multiple choices from which they should select the proper hyponyms are comparatively low in the pre-test. During the post-test, the participants were asked to write the accurate hyponyms in the blanks without any choices from the definition of each hyponyms. The participants were expected to write the following hyponyms by using the knowledge gained in WordNet teaching. The hyponyms are Ant, Autism, Battery, Carbon, Dolphin, Einstein, Fuel, Tag, Video and Website. The mean and median values of the post-test are 32.2 and 32.5 respectively. Participants' knowledge about the hyponyms of the nouns were evaluated and recorded. The results revealed that participants' knowledge and vocabulary acquisition has significantly increased and the effective use of WordNet has proved as advancements towards the ESL learners' based teaching pedagogy.

\section{Conclusions}

The current experimental research may not solve all the issues on English language and improvements but it aims at addressing the major problems to bring up the ESL learners community in qualitative levels of vocabulary acquisition.
TED specific versions were found to be the advancement towards AWL or GSL. Hence it was suggested that more course on efficient learning may pay a better way for the successful and improved vocabulary abilities. Students need to get trained through additional courses on vocabulary development. WordNet would be a useful and effective electronic database of English to learn vocabulary knowledge especially hyponyms. The current study focused on teaching learners how to use the proper hyponyms of a noun. To that end, a new teaching method might draw on both the TED wordlist noun corpora and WordNet is experimented well in the study.

\section{REFERENCES}

[1] Alqahtani, M. (2015). “The importance of vocabulary in language learning and how to be taught”. International Journal of Teaching and Education. III (3). pp. 21-34.

[2] Aravind, B. R., \& Rajasekaran, V. (2019). “Technological modality to influence persuasive and argumentative vocabulary for effective communication with reference to selected TED talk videos”. International Journal of Recent Technology and Engineering, 7(5), 165-170.

[3] Aravind, B. R., \& Rajasekaran, V. (2018). “Advanced technological modality to explore ESL learners' vocabulary knowledge through social strategies”. Journal of Advanced Research in Dynamical and Control Systems, 10(10), 250-256.

[4] Atkinson, R. C., \& Raugh, M. R. (1975). “An application of the mnemonic keyword method to the acquisition of a Russian vocabulary”. Journal of Experimental Psychology: 
Human Learning and Memory, 104, 126-133.

[5] Beck, I. L., McKeown, M. G., \& Omanson, R. C. (1987). "The effects and uses of diverse vocabulary instructional techniques”. In M. G. McKeown \& M. E. Curtis (Eds.), The nature of vocabulary acquisition (pp. 147-163).

[6] Carter, R. (2012). "Vocabulary: Applied linguistics perspectives”. Routledge.

[7] Hu, X., Graesser, A. C., \& Tutoring Research Group.(1998). "Using WordNet and latent semantic analysis to evaluate the conversational contributions of learners in the tutorial dialog”. In Proceedings of the international conference on computers in education (Vol. 2, pp. 337-341).

[8] Kamil, M. \& Hiebert, E.H. (2005). "The teaching and learning of vocabulary: Perspectives and persistent issues. In: Teaching and learning vocabulary: Bringing scientific research to practice”. [Online]. Mahwah, NJ: Erlbaum. Lin, C. C. (1997). "Semantic network for vocabulary teaching". Journal of Taiwan Normal University: Humanities \& Social Science, 42, 43-54.

[9] Mandala, Rila, Tokunaga, T., Tanaka, Hozumi, O., Akitoshi, Satoh, K. (1998). “Ad Hoc Retrieval Experiments Using WordNet and Automatically Constructed Thesauri”. TREC-7; 414-419.

[10] Massing, C., Kirova, A. \& Hennig, K. (2013). “The Role of First Language Facilitators in Redefining Parent Involvement: Newcomer Families' Funds of Knowledge in an Intercultural Preschool Program”. Journal of Childhood Studies. 38 (2).

[11] Moldovan, D.I. and Mihalcea, R. (2000). "Using WordNet and lexical operators to improve Internet searchers". IEEE Internet Computing, 4 (1); 34-43.

[12] Ramanayake, S. \& Williams, C. (2017). “I Don’t Know Why I'm Learning This: Preservice English Teachers 'Engagement in a Language Development Course". International Journal of Teaching and Learning in Higher Education, 29 (3). pp. 447-457.

[13] Resnick, P (1993). "Selection and Information: A class-based approach to lexical relationships". PhD dissertation.University of Pennsylvania (1993).

[14] Ritchie, J. \& Lewis, J. (2003). “Qualitative Research Practice: A Guide for Social Science Students and Researchers”. New Delhi: SAGE Publications.

[15] Roe, K.L. (2016). "Cultural Relevance in an English Language Learners' Classroom: A Qualitative Case Study”. Walden University.

[16] Sternberg, R. J. (1987). "Most vocabulary is learned from context”. In M. G. McKeown \& M. E. Curtis (Eds.), The nature of vocabulary acquisition (pp. 89-105). Hillsdale, NJ: Lawrence Erlbaum. 